\title{
APLICAÇÃO DO MÉTODO ELETROMAGNÉTICO INDUTIVO (EM) NO MONITORAMENTO DE CONTAMINANTES EM SUBSUPERFÍCIE
}

\author{
César Augusto Moreira ${ }^{1}$, Wagner França Aquino² e João Carlos Dourado ${ }^{3}$ \\ Recebido em 19 abril, 2006 / Aceito em 25 outubro, 2006 \\ Received on April 19, 2006 / Accepted on October 25, 2006
}

\begin{abstract}
The combined use of monitoring wells and geophysical techniques constitutes the form most adequate for identification and the monitoring contaminated areas. This procedure allows the location of wells and monitoring in strategically points, optimizing the results and minimizing costs. The Electromagnetic Inductive Method (EMI) presents ample possibilities of application in environmental studies due the facility in the acquisition of data, versatility of the equipment in field and the possibility of scanning in the ample areas in short time. This paper realizes a comparative analysis of EMI data collected in 1992 and 2003. The studied place is an industrial area that presents contamination the soil and groundwater for Benzene, Toluene, Xylene, 1,2 dichloroethene, Sodium and Chloride, product of the infiltration of effluent chemistries directly of the ground. The results indicate accented attenuation of the contaminant plume, with probable reduction in the contamination degree. The composites of light non-aqueous phase liquids (LNAPLS) had presented superior trend of horizontal, concomitant migration to the movement of the groundwater. The composites of dense non-aqueous phase liquids (DNAPLs) had more presented an accented trend of migration in the vertical direction, possibly due to absence of impermeable surfaces. The inorganics composites had followed the flow of composites of non-aqueous phase liquids (NAPLs), what in turn it allowed the characterization of the contamination plume as a conductive body in relation to the adjacent areas.
\end{abstract}

Keywords: plume of contamination, NAPL, natural attenuation, electromagnetic inductive, organochloride.

RESUMO. 0 uso combinado de poços de amostragem e técnicas geofísicas constitui a maneira mais adequada para identificação e 0 monitoramento de áreas contaminadas. Este procedimento permite a locação de poços de amostragem e de monitoramento em pontos estratégicos, otimizando resultados e minimizando gastos. 0 método geofísico Eletromagnético Indutivo (EM) apresenta amplas possibilidades de aplicação em estudos ambientais devido à facilidade na aquisição de dados, versatilidade do equipamento em campo e a possibilidade de varredura de grandes áreas num curto espaço de tempo. Este trabalho realiza uma análise comparativa de dados EM obtidos no ano de 1992 e em 2003. 0 local estudado é uma área industrial que apresenta contaminação do solo e água subterrânea por Benzeno, Tolueno, Xileno, 1,2 dicloroetano, Sódio e Cloreto, produto da infiltração de efluentes químicos diretamente do solo. Os resultados indicam uma acentuada atenuação da pluma contaminante, com provável redução no grau de contaminação. Os compostos de fase leve (LNAPLs) apresentaram maior tendência de migração horizontal, concomitante ao movimento da água subterrânea. Os compostos de fase densa (DNAPLs) apresentaram uma tendência mais acentuada de migração no sentido vertical, possivelmente devido à ausência de superfícies impermeáveis. Os compostos inorgânicos acompanharam o fluxo dos compostos de fase líquida não aquosa (NAPLs), 0 que por sua vez permitiu a caracterização da pluma de contaminação como um corpo condutivo em relação às áreas adjacentes.

Palavras-chave: pluma de contaminação, NAPL, atenuação natural, Eletromagnético Indutivo, organoclorado.

\footnotetext{
${ }_{1}^{1}$ IGCE, UNESP, Campus de Rio Claro, Av. 24-A, 1515, Bela Vista, Caixa Postal 178, 13506-900 Rio Claro, SP, Brasil - E-mail: cesargeologi@@yahoo.com.br

${ }^{2}$ GPR Geoscience Geofísica Ltda., Rua Mazel, 174, Ioja 3, Parque São George, 06708-235 Cotia, SP, Brasil - E-mail: wagnergpr@terra.com.br

3 Departamento de Geologia Aplicada, IGCE, UNESP, Campus de Rio Claro, Av. 24-A, 1515, Bela Vista, Caixa Postal 178, 13506-900 Rio Claro, SP, Brasil -E-mail: jdourado@rc.unesp.br
} 


\section{INTRODUÇÃo}

0 crescente processo de industrialização e criação de novas tecnologias resulta na geração de empregos diretos e indiretos, aumento na arrecadação de impostos, e em última análise, aumento da qualidade e expectativa de vida.

Todo processo industrial produz resíduos ou efluentes, que pode variar grau de toxicidade em razão dos tipos de compostos utilizados, grau de refinamento do processo, complexidade, entre outros. A destinação final destes produtos tem sido uma preocupação constante e crescente, pois a cada ano aumenta a quantidade de resíduos e efluentes gerados.

0 descarte inadequado e ausente de quaisquer controles tem causado danos ao meio ambiente e a saúde humana. Em muitos casos, esta medida é tomada por causa do elevado custo de reciclagem ou reutilização destes produtos. Contudo, este é um procedimento criminoso e está sujeito a penalidade tanto por parte dos órgãos ambientais quanto por ações civis.

A identificação e o diagnóstico de áreas contaminadas por efluentes líquidos é um procedimento necessário afim de que seja possível tomar medidas de contenção da fonte poluidora e posterior remediação.

0 uso combinado de poços de amostragem e técnicas geofísicas constitui uma das maneiras mais adequada para identificação e o monitoramento de áreas contaminadas. Este procedimento permite a locação de poços de amostragem e monitoramento nos pontos adequados, que por sua vez impede o estabelecimento de um número excessivo destes poços.

A rapidez, precisão, versatilidade e 0 custo relativamente baixo, também permitem aplicações de técnicas geofísicas nas etapas de remediação e monitoramento das áreas previamente diagnosticadas.

A aplicação do método Eletromagnético Indutivo (EM) em estudos ambientais reside na possibilidade de execução de leituras de condutividade aparente do terreno para diversas profundidades. As principais vantagens desta técnica geofísica são a facilidade de aquisição de dados, realização de leituras em diversas profundidades, versatilidade do equipamento em campo e a possibilidade de varredura de grandes áreas num curto espaço de tempo (McNeill, 1980a; EPA, 1993; Goldstein et al., 1990). Estas vantagens são traduzidas em rapidez e baixo custo.

A principal desvantagem desta técnica é o produto da interação do campo eletromagnético gerado pelo equipamento, com estruturas metálicas, fios de alta tensão tambores metálicos e outras estruturas (McNeill, 1980a). Esta interação resultada em valores de condutividade aparente muito superiores aos valores reais. Desta forma, esta técnica pode ter seu uso limitado em centros urbanos, instalações industriais, entre outros.

Este trabalho apresenta os resultados da aplicação no método Eletromagnético Indutivo em uma área industrial abandonada, onde foi diagnosticada a contaminação do solo e da água subterrânea por Benzeno, Tolueno, Xileno (BTX), 1,2 dicloroetano e compostos inorgânicos. A aplicação do método envolveu duas ocasiões, a primeira em 1992, pouco tempo após o diagnóstico da área, e a segunda em 2003, para avaliação do processo de atenuação natural da pluma de contaminação.

\section{MATERIAIS E MÉTODOS Histórico da área de estudos}

A área está localizada no distrito industrial do município de Araras, estado de São Paulo, Brasil (Fig. 1). O local foi ocupado por duas indústrias químicas entre 1981 e 1992. Dentre os produtos fabricados estão o dieloxalato, acetato de etila, 4-amino-N2-tiozolil-benzeno-sulfonamida, acetopiruvato de metila, 5-metil3-carboxiamidoxazol, e dentre os solventes utilizados durante 0 processo eram estão o 1,2 dicloroetano e o tolueno (CETESB, 1997). Os solventes eram reutilizados após passarem por colunas de destilação, e os efluentes gerados eram infiltrados diretamente no solo, em dois poços presentes na área. Este procedimento foi utilizado entre 1981 e 1988 (CETESB, 1997).

Em 1988 foi constatada a contaminação do solo e da água subterrânea por 1,2 dicloroetano, benzeno, tolueno, xileno, sódio e cloreto. A partir de 1990 foi dado início ao monitoramento da contaminação pela Agência de Proteção Ambiental do Estado de São Paulo (CETESB), por meio de diversos poços de monitoramento instalados na área industrial e adjacências.

No ano de 1992, Aquino (2000) iniciou estudos nesta área a partir da aplicação das técnicas geofísicas de Radar de Penetração no Solo (GPR) e o método Eletromagnético Indutivo (EM). Em 1997 a CETESB realizou novo levantamento geofísico com a aplicação dos métodos de Eletrorresistividade e EM.

Em estudo para avaliação do processo de atenuação natural, Moreira (2003) realizou novo diagnóstico da área contaminada a partir da avaliação dos dados de análises químicas da água subterrânea e aplicação das técnicas de GPR e EM no ano de 2003.

\section{Geologia e hidrogeologia}

0 meio geológico da área contaminada é caracterizado por siltito argiloso pertencente à formação Tatuí, com espessura local em torno de 60 metros. 0 produto de alteração desta rocha é representado por solo vermelho escuro argiloso, com matriz de fração 


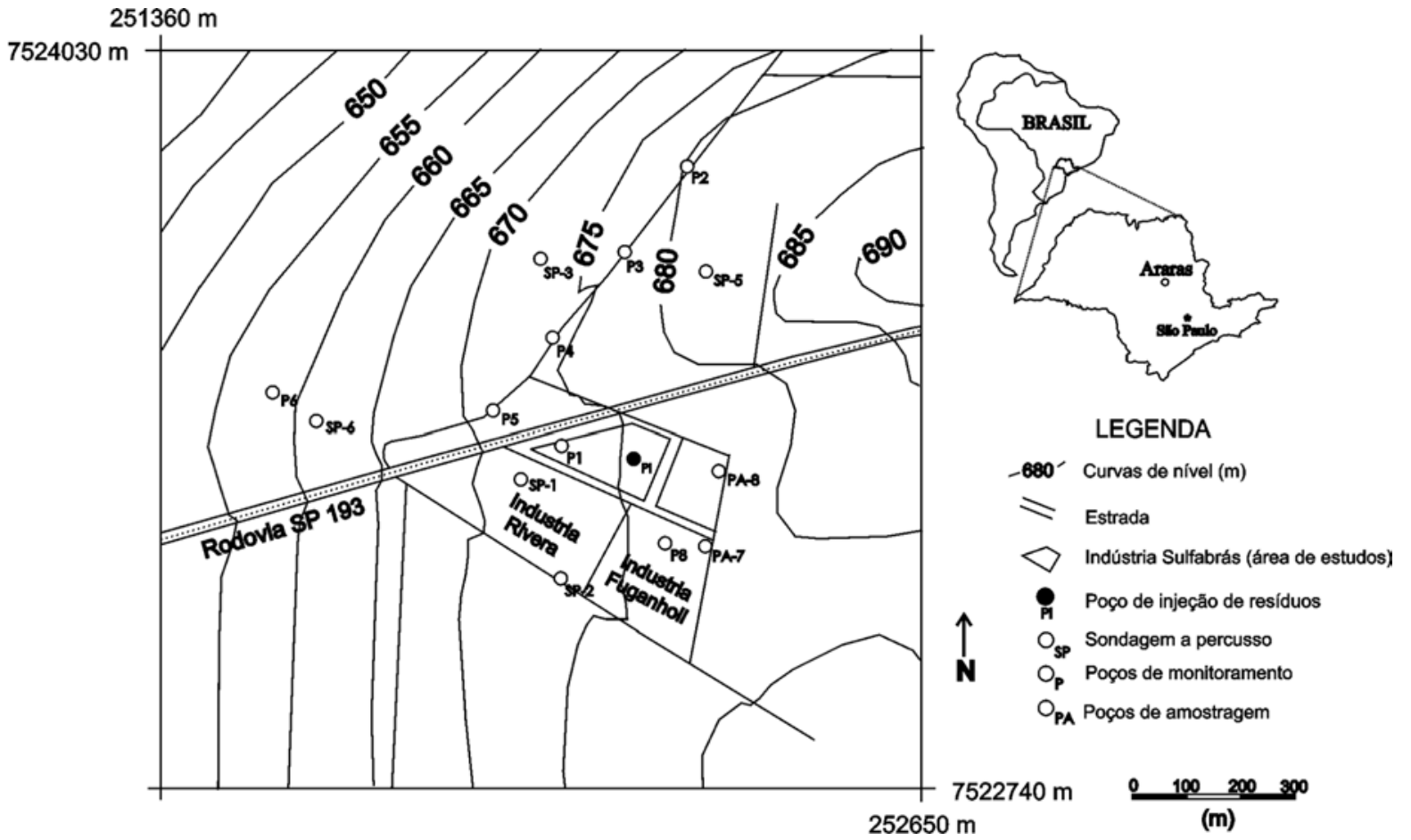

Figura 1 - Mapa de localização.

silte entre 1 e 3 metros de profundidade e com matriz de fração argila entre 3 a 13 metros de profundidade, com transição gradual entre os níveis.

A formação Tatuí é sotoposta por uma ampla gama de litotipos, que variam de arenitos porosos a calcários silicificados, reunidos no Grupo Itararé. Este grupo representa um importante aqüifero regional, denominado Aqüífero Tubarão, responsável por parte do abastecimento de uma das regiões mais densamente povoadas e industrializadas do Brasil.

0 nível freático local está situado em profundidades entre 10,7 a 14,1 metros, com variações sazonais entre 7,9 a 14,9 metros. 0 fluxo da água subterrânea nas proximidades dos pontos de infiltração de resíduos é para sudoeste. A condutividade hidráulica varia de 4,4 × $10^{-7}$ a 7,0 × 10-7 (CETESB, 1997; Aquino, 2000).

\section{Caracterização dos Compostos Orgânicos}

Os compostos orgânicos que constituem a pluma de contaminação são denominados de fase líquida não aquosa (NAPLS), cujas diferenças nas propriedades físicas e químicas resultam na imiscibilidade em água e no ar (Newell et al., 1995). Quanto à densidade, os compostos de NAPLs podem ser divididos em duas classes:
- Compostos de Fase Líquida Leve Não Aquosa - LNAPL, caracterizados por serem mais leves que a água. Tais compostos são representados na área contaminada por benzeno, tolueno e xileno.

- Compostos de Fase Líquida Densa Não Aquosa - DNAPL, caracterizados por serem mais densos que a água. Esta fase contaminante é representada na área por 1, 2 dicloroetano.

Após a liberação no ambiente, os compostos de LNAPLs tendem a se a infiltrar no solo por ação da força da gravidade. Ao atingir a franja capilar, os compostos de LNAPL passam a ser mover lateralmente, sob a forma de fase livre ao longo da camada superior da zona saturada devido à ação da gravidade e de forças de capilaridade (Newell et al., 1995).

A migração de LNAPL em subsuperfície é controlada por chuvas, variações sazonais e pelo gradiente hidrogeológico, associada ao fluxo d’água subterrânea (Daniels et al., 1994).

Os compostos de DNAPL tendem a migrar pelo interior da zona saturada por ação da gravidade, e eventualmente é exaurido por processos de saturação residual. Contudo, caso este processo não consuma o DNAPL completamente, o composto continuará a migrar verticalmente até ser retido em zonas de baixa permeabilidade (Huling \& Weaver, 1991). 


\section{Método Eletromagnético Indutivo}

0 método Eletromagnético Indutivo mede a condutividade elétrica em subsuperfície a partir da técnica de baixo número de induções (McNeill, 1980a).

Este método tem como princípio a passagem de uma corrente elétrica alternada por meio de uma bobina emissora, que resulta em um campo eletromagnético primário. A propagação deste campo em um meio condutor no subsolo, assumido como meio homogêneo, resulta em correntes secundárias alternadas que produzem um campo eletromagnético secundário (Fig. 2).

Uma bobina receptora posicionada dentro da área de influência dos campos primário e secundário capta a corrente induzida resultante da soma dos campos gerados. As diferenças de intensidade, direção e fase entre os campos eletromagnético primário e secundário podem revelar a presença de corpos condutores (McNeill, 1980a).

Geralmente 0 campo secundário é uma função complexa da distância entre bobinas $(s)$, freqüência de operação $(f)$ e condutividade $(\sigma)$ do meio. Sob determinadas restrições, tecnicamente definidas como operação a baixo número de induções, 0 campo magnético secundário é uma função bastante simples destas variáveis. Tais restrições são incorporadas no condutivímetro EM 34-3, onde o campo magnético secundário é definido como:

$$
\frac{H p}{H s} \simeq \frac{I \omega \mu_{o} \sigma S^{2}}{4}
$$

sendo $H p$ o campo magnético primário, Hs o campo magnético secundário, $o=2 \pi f, f=$ freqüência em hertz, $\mu_{0}=$ permeabilidade no espaço vazio, $\sigma=$ condutividade do meio (mho/m), $S=$ espaçamento entre bobinas e $I=\sqrt{-1}$.

Os parâmetros envolvidos na propagação de ondas eletromagnéticas são a condutividade elétrica $(\sigma)$ e a permissividade dielétrica $(K)$ dos materiais de subsuperfície. A condutividade elétrica varia em função do tipo de solo ou rocha, porosidade, permeabilidade, grau de saturação e em função das propriedades eletroquímicas dos fluídos de preenchimento de poros, este último apresenta-se como fator dominante em muitos casos (Sheriff, 1989; McNeill, 1980b).

A razão entre campo magnético secundário e primário é admitida como linearmente proporcional à condutividade do meio, premissa que permite leituras diretas a partir da simples medida desta razão.

0 equipamento utilizado foi o condutivímetro EM 34-3 modelo XL, fabricado pela Geonics Ltd. - Canadá. Este equipamento é calibrado para leituras diretas de condutividade aparente por meio da expressão:

$$
\sigma_{a}=\frac{4}{(2 \pi f) \mu s^{2}}\left(\frac{H s}{H p}\right)
$$

sendo $H s / H p$ a razão entre o campo magnético secundário $(H s)$ e o campo magnético primário $(H p) ; \sigma_{a}$ é a condutividade aparente, em siemens por metro; $\mu$ é a permeabilidade magnética, em hertz por metro; $f$ é a freqüência em hertz e $s$ é o espaçamento entre as bobinas, em metros.

A variação na distância e na posição das bobinas permite a leitura da condutividade aparente para diversas profundidades. Neste trabalho foram utilizados os cabos de referência de 10 e 40 metros, que em conjunto com a variação na posição das bobinas permitiu leituras em quatro profundidades (Tab. 1).

Tabela 1 - Profundidade de exploração do EM 34-3 com variação no espaçamento entre as bobinas (McNeill, 1980a).

\begin{tabular}{|c|c|c|}
\hline $\begin{array}{c}\text { Espaçamento } \\
\text { entre bobinas }(\mathrm{m})\end{array}$ & Posição & $\begin{array}{c}\text { Profundidade } \\
\text { de exploração }(\mathrm{m})\end{array}$ \\
\hline \multirow{2}{*}{10} & vertical & 7,5 \\
& horizontal & 15 \\
\hline \multirow{2}{*}{40} & vertical & 30 \\
& horizontal & 60 \\
\hline
\end{tabular}

\section{Aquisição e processamento}

Tanto os dados adquiridos neste trabalho como os dados de Aquino (2000), foram adquiridos por meio da técnica de caminhamento eletromagnético (Greenhouse \& Slaine, 1986), com espaçamento médio de 20 metros entre pontos de leitura.

As linhas de caminhamento eletromagnético foram definidas com base em análises químicas da água subterrânea, levantamentos geofísicos preliminares e nos acessos disponíveis. Foram realizados 160 pontos de leituras de condutividade aparente para cada uma das profundidades máximas de investigação possíveis para o equipamento utilizado (Tab. 1).

Os dados foram integrados por interpolação dos valores a partir do programa SURFER, versão 8, desenvolvido pela Golden Software - EUA. Durante o processamento foram excluídos alguns pontos de leitura, por apresentarem valores discrepantes, muitos dos quais já haviam sido previamente reconhecidos em campo, como transformadores elétricos, cruzamento de linhas de alta tensão, motores elétricos em funcionamento, dentre outros.

\section{RESULTADOS}

A análise dos mapas de condutividade aparente indica que a pluma de contaminação atinge não somente a área da indústria, 


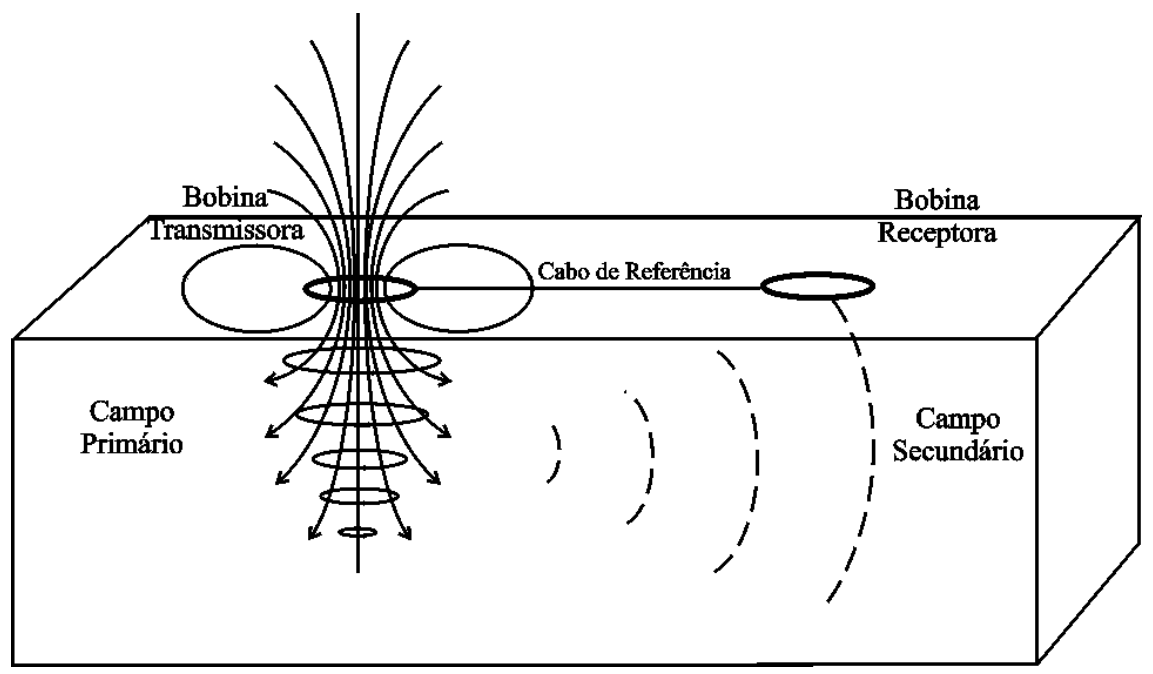

Figura 2 - Princípio do Método Eletromagnético Indutivo.

mas também 0 subsolo de áreas adjacentes (Figs. 3 e 4). Contudo, as áreas com maior concentração de contaminante estão restritas a área da indústria Sulfabrás.

Os pontos de maior condutividade em praticamente todos os mapas estão associados ao poço de injeção de efluentes, exceto no mapa para $60 \mathrm{~m}$ de profundidade de 1992 (Fig. 4F).

Houve aumento nas dimensões da pluma de contaminação no intervalo de até $15 \mathrm{~m}$ de profundidade e uma redução significativa nos valores de condutividade aparente nas áreas próximas ao poço de infiltração, principalmente valores acima de $30 \mathrm{~ms} / \mathrm{m}$ (Figs. 3A, 3B, 3C e 3D).

A redução nos valores de condutividade aparente está diretamente associada á redução nos teores relativos de contaminação para 0 intervalo de até $15 \mathrm{~m}$ de profundidade. A ausência de infiltrações durante 0 intervalo de tempo analisado, decomposição dos contaminantes, diluição dos teores de contaminação e migração vertical dos contaminantes DNAPL, estão entre os principais fatores que contribuíram para esta redução.

0 aumento nas dimensões da pluma de contaminação neste intervalo de profundidade indica migração horizontal dos contaminantes LNAPL concomitante ao fluxo da água subterrânea, seja sob a forma dissolvida ou como fase livre.

Os mapas para as profundidades de 30 e 60 metros (Figs. $4 A, 4 B, 4 C, 4 D$ ), revelam que houve um pequeno aumento nas dimensões da pluma de contaminação, concomitante aos valores relativos de condutividade aparente. Este fato está diretamente associado ao aumento nos teores de contaminação, mesmo com 0 término de injeção de efluentes.
Este aumento está associado ao comportamento dos compostos DNAPL, caracterizados pela elevada densidade relativa. Desta forma, tais compostos tendem a migrar no sentido vertical, levando consigo compostos inorgânicos dissolvidos.

\section{DISCUSSÃO}

A detecção de anomalia de alta condutividade em profundidade foi possível devido à presença de compostos inorgânicos - sódio e cloreto - associados a frações degradadas dos compostos orgânicos, cujo produto resultante possui condutividade elétrica menor que o meio geológico. Nobes (1996) relata que a presença de contaminantes na zona saturada altera a condutância do meio, que resulta em contraste com o meio em redor não contaminado.

0 corpo de alta condutividade detectado no ensaio geofísico pode dividido em dois estratos, separados pelo nível freático. 0 estrato superior compreende a zona não saturada e saturada, até a profundidade de 15 metros. 0 estrato inferior corresponde à zona saturada e sua base está situada abaixo de 60 metros, visto que a partir da técnica geofísica aplicada, não foi possível determinar a profundidade da base deste corpo.

Os contaminantes orgânicos que constituem o estrato superior do corpo condutivo são os compostos LNAPL, associados aos íns inorgânicos dissolvidos. Os compostos LNAPL, caracterizados pela baixa densidade relativa, apresentam migração associada ao fluxo da água subterrânea. A redução nos valores de condutividade aparente neste estrato durante o período analisado (Figs. 3A, 3B, 3C, 3D), indica dispersão, diluição de ambas as classes de contaminação e extinção da fonte contaminadora. 

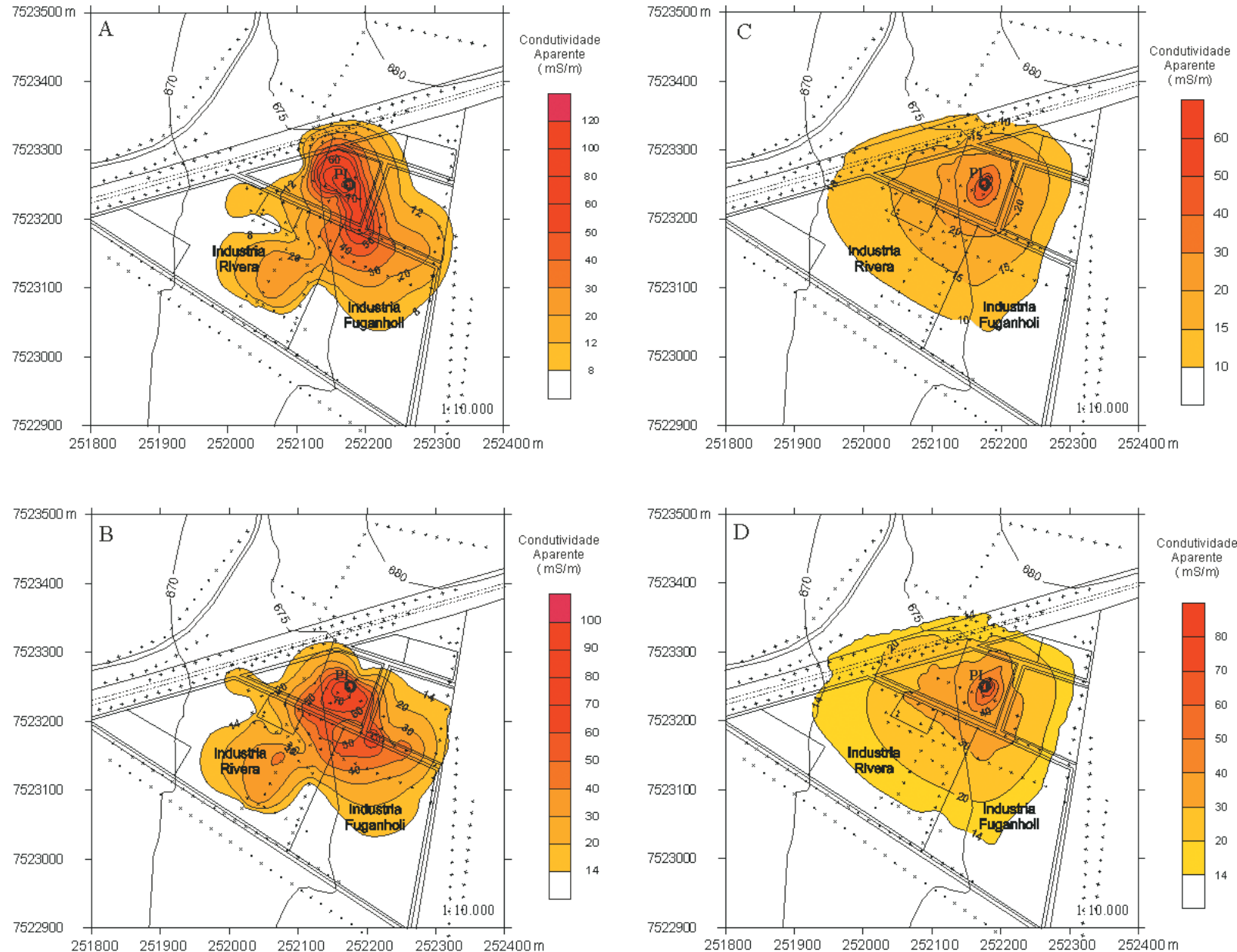

$\times$ Pontos de Leitura

PJ Poço de infiltração de resíduos

Figura 3 - Mapas de isocondutividade aparente. Profundidade de até 7,5 m (A), $15 \mathrm{~m}$ (B) em 1992 e profundidade de até 7,5 m (C) e 15 m (D) em 2003.

0 estrato inferior do corpo condutivo é constituído de contaminantes orgânicos DNAPL, também associados aos compostos inorgânicos dissolvidos. A aparente dissociação entre o ponto de injeção de efluentes e as anomalias de condutividade no mapa para 60 metros de 1992 (Fig. 4B), pode indicar um período em que os compostos DNAPL estavam com pequenas quantidades de sais inorgânicos. Desta forma, os compostos DNAPL presentes não puderam ser detectados a partir do método Eletromagnético Indutivo.

\section{CONCLUSÕES}

0 método Eletromagnético Indutivo foi capaz de detectar uma anomalia de alta condutividade aparente na área estudada, associada com o ponto de injeção de efluentes, nas duas ocasiões em que foi utilizado. Os dados de condutividade aparente são valores meramente qualitativos, junto aos quais é indispensável à realização de análises químicas para identificação de contaminantes eventualmente presentes.

Entretanto, os mapas produzidos a partir dos valores de condutividade aparente apresentam claramente a dinâmica da pluma de contaminação e a tendência de fluxo de seus constituintes. Desta forma, o método Eletromagnético Indutivo demonstra ser uma ferramenta eficiente para o monitoramento de áreas contaminadas por compostos condutivos.

A caracterização desta anomalia, a partir das análises químicas da água subterrânea, indica que o caráter condutivo do corpo contaminante é resultado da presença de compostos inorgânicos dissolvidos, associados a compostos orgânicos (NAPLs) degradados, dissolvidos ou diluídos. 

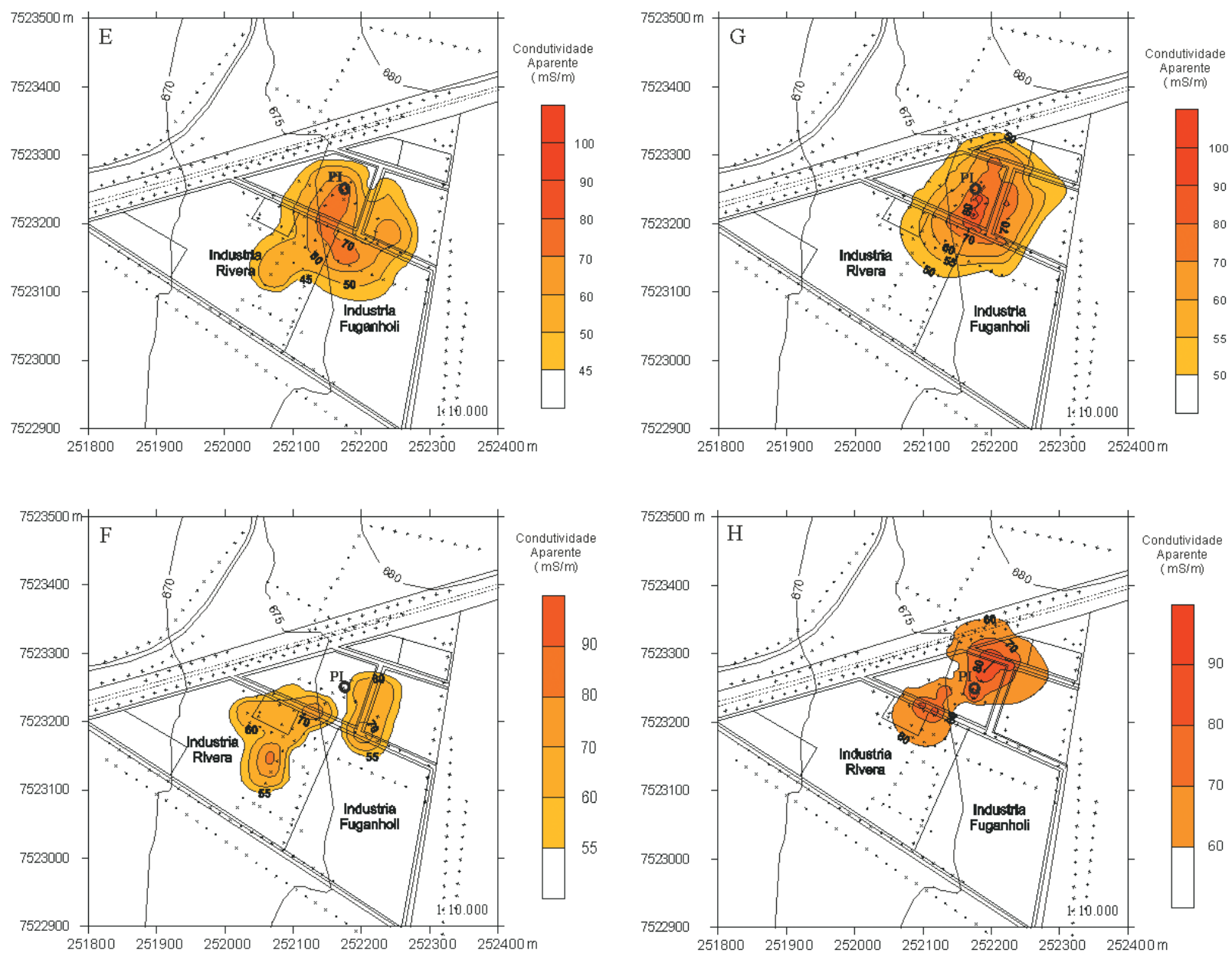

$x_{x}$ Pontos de Leitura

PJ Poço de infiltração de resíduos

Figura 4 - Mapas de isocondutividade aparente. Profundidade de até $30 \mathrm{~m}$ (E) e $60 \mathrm{~m}$ (F) em 1992 e profundidade de até $30 \mathrm{~m}$ (G) e $60 \mathrm{~m}$ (H) em 2003

0 monitoramento da pluma contaminante por meio de ensaios geofísicos em vários períodos, revela o comportamento dos compostos constituintes, frente a dinâmica do meio geológico. A ação de forças como gravidade, capilaridade, diluiçãa, dispersão, degradação, entre outras, governam o fluxo dos contaminantes orgânicos em sentidos completamente opostos. Contudo, descrevem um comportamento esperado e previsto em diversos trabalhos (Daniels et al., 1994; Benson et al., 1997; Benson \& Stubben, 1995; Sauck, 2000; Atekwana et al., 2000; Meju, 2000; Aristodemou \& Thomas-Betts, 2000).

\section{AGRADECIMENTOS}

Este trabalho foi realizado com o apoio da Agência Nacional do Petróleo, Gás Natural e Biocombustíveis - ANP e da Financiadora de Estudos e Projetos - FINEP, por meio do Programa de
Recursos Humanos para o Setor de Petróleo e Gás - PRH-05ANP/MCT. Os autores agradecem ao técnico Francisco Manuel Garcia Barrera, do Departamento de Geologia Aplicada, UNESP - Rio Claro, pelo auxílio na execução dos trabalhos de campo. Agradecemos também ao Instituto Astronômico e Geofísico IAG/USP pelo empréstimo do equipamento geofííico.

\section{REFERÊNCIAS}

AQUINO WF. 2000. Métodos Geofísicos Eletromagnéticos aplicados ao diagnóstico da contaminação de Solo e das Águas Subterrâneas em área de Infiltração de Resíduos Industriais. 121f., Dissertação, Universidade de São Paulo.

ARISTODEMOU E \& THOMAS-BETTS A. 2000. DC resistivity and induced polarisation investigations at a waste disposal site and its environments. Journal of Applied Geophysics, 44: 275-302. 
ATEKWANA EA, SAUCK WA \& WERKEMA JR DD. 2000. Investigations of geoelectrical signatures at a hydrocarbon contaminated site. Journal of Applied Geophysics, 44: 167-180.

BENSON AK \& STUBBEN MA. 1995. Interval resistivities and very low frequency electromagnetic induction - an aid to detecting groundwater contamination in space and time: a case study. Environmental Geosciences, 2: 74-84.

BENSON AK, PAYNE KL \& STUBBEN MA. 1997. Mapping groundwater contamination using DC resistivity and VLF geophysical methods a case study. Geophysics, 62(1): 80-86

CETESB. Companhia de Tecnologia de Saneamento Ambiental. 1997. Relatório do levantamento geofísico para diagnóstico da contaminação da indústria Sulfabrás, CETESB, São Paulo, 91 pp.

DANIELS JJ, ROBERTS R \& VENDL M. 1994. Ground Penetrating Radar for the detection of liquid contaminants. Journal of Applied Geophysics, 33: 195-207.

EPA. Environmental Protection Agency. 1993. Use of Airborne, Surface and Borehole Geophysical Techniques at Contaminated Sites. Washington, chapter 1: 4-6.

GOLDSTEIN NE, BENSON SM \& ALUMBAUGH D. 1990. Saline Groundwater Plume Mapping with Electromagnetics. Geotechnical and Environmental Geophysics, Investigations in Geophysics, S.E.G., II(5): $17-27$.

GREENHOUSE JP \& SLAINE DD. 1986. Geophysical modelling and mapping of contaminated groundwater around three waste disposal sites in southern Ontario. Canadian Geotechnical Journal, 23: 372-384.
HULING SG \& WEAVER JW. 1991. Dense Nonaqueous Phase Liquids. Ground Water Issue, Environmental Protection Agency, Oklahoma, 21 pp.

McNEILL J. 1980a. Electromagnetic Terrain Conductivity Measurement at Low Induction Numbers. Technical Note TN, 6: 6-15. Disponível em: $<$ http://www.geonics.com>. Acesso em: 22 maio 2003.

McNEILL J. 1980b. Electrical Conductivity of Soils and Rocks. Technical Note TN, 5: 21. Disponível em: <http://www.geonics.com>. Acesso em: 22 maio 2003.

MEJU MA. 2000. Geoelectrical investigation of old/abandoned, covered landfill sites in urban areas: model development with a genetic diagnosis approach. Journal of Applied Geophysics, 44: 115-150.

MOREIRA CA. 2003. Análise Temporal e Espacial de Pluma de Contaminação por meio de métodos geofísicos, monografia de Trabalho de Conclusão de Curso, Universidade Estadual Paulista - UNESP 84f.

NEWELL CJ, ACREE SD, ROSS RR \& HULING SG. 1995. Light Nonaqueous Phase Liquids. Ground Water Issue, Environmental Protection Agency, Oklahoma, 28 pp.

NOBES DC. 1996. Troubled Waters: environmental applications of electrical and electromagnetic methods. Surveys in Geophysics, 17: 393-454.

SAUCK WA. 2000. A model for the resistivity structure of LNAPL plumes and their environs in sandy sediments. Journal of Applied Geophysics, 44: $151-165$

SHERIFF RE. 1989. Geophysical Methods, Prentice Hall, New Jersey, pp. 175-201.

\section{NOTAS SOBRE OS AUTORES}

César Augusto Moreira. Bacharel em Geologia pelo Instituto de Geociências e Ciências Exatas (IGCE) da Universidade Estadual Paulista (UNESP) em 2003. Trabalhou como geólogo de mina na Santa Elina Desenvolvimento Mineral (Yamana Gold Inc.) e concluiu mestrado em Geociências e Meio Ambiente em 2005. Atualmente é aluno de doutorado no programa de Pós-Graduação em Geociências e Meio Ambiente do Instituto de Geociências e Ciências Exatas, UNESP, Campus de Rio Claro.

Wagner França Aquino. Bacharel em Geofísica pelo Instituto Astronômico e Geofísico (IAG) (1989), Mestre em Recursos Minerais e Hidrogeologia pelo Instituto de Geociências (IG) (2000), Universidade de São Paulo (USP). Trabalhou no setor de áreas contaminadas na Companhia de Tecnologia e Saneamento Ambiental (CETESB) (1992-2006). Atuou como consultor técnico da Bureau Veritas (2006). Atualmente é Sócio-diretor da empresa GPR Geoscience Geofísica Ltda.

João Carlos Dourado. Bacharel em Geologia pelo Instituto de Geociências da Universidade de São Paulo (USP) em 1977. Doutor em Geociências pela UNESP em 1997. Trabalhou com geofísica aplicada no Instituto de Pesquisas Tecnológicas do Estado de São Paulo de 1977 a 1997. Atualmente é professor assistente doutor do Departamento de Geologia Aplicada do IGCE-UNESP onde atua desde 1997. Trabalha com métodos geofísicos aplicados à Hidrogeologia, Meio Ambiente, Geologia de Engenharia e Geotectônica. 